\title{
Dynamic UMTS Simulator for Congestion Studies and Evaluation of Resource Management Techniques
}

\author{
Sami Nousiainen, Krzysztof Kordybach, Paul Kemppi, Veli-Pekka Kröger \\ VTT Information Technology, Tekniikantie 4B, FIN-02044 Espoo, Finland \\ Tel: +35894564415, Fax: +3589456 6027, Email: sami.nousiainen@vtt.fi
}

\begin{abstract}
A dynamic UMTS system level simulator has been designed and implemented in Windows NT/2000 environment using Visual C++. The objective has been to design and implement such a simulator that can be used to create and study different traffic load scenarios and resource management techniques for tackling congestion situations.
\end{abstract}

An UMTS system level simulator that is comprehensive and flexible and takes into consideration 3GPP recommendations is presented in this paper. It contains very detailed models of many important UMTS features. Simulator features designed for creating different load situations in the network are described. Furthermore, a congestion/admission control framework for UMTS is presented. Finally, simulation results illustrating the congestion causation features and congestion detection are shown.

Key words: UMTS, simulator, congestion, resource management

\section{INTRODUCTION AND BACKGROUND}

The operation and resource management in the air interface of CDMA based networks, such as UMTS, is considerably more difficult than in networks employing FDMA/TDMA technology, such as GSM. Accurate system level simulations are required for the assessment of the network performance under different conditions. Some previous studies related to load control and system level simulators can be found in [1], [2], [3], [4], [5], [7].

\footnotetext{
The original version of this chapter was revised: The copyright line was incorrect. This has been corrected. The Erratum to this chapter is available at DOI: 10.1007/978-0-387-35618-1_37
} 


\section{STRUCTURE AND MODULES OF THE IMPLEMENTED SIMULATOR}

Cells and UEs. Each cell has e.g. sector number, neighbors, TX power limitation and antenna coordinates. Each UE has e.g. momentary TX power, coordinates, speed, direction, mobility class, service type, QoS level, terminal type and access class.

Channel model. Common pilot channel (CPICH) transmitted in the downlink direction is modeled in the simulator. It serves as a reference for e.g. cell selection and soft handover algorithms. In addition to that, dedicated channels with data and control parts are modeled in the uplink and downlink directions. Power offset between the data and control parts can be given separately for each service type. The data part of the dedicated channel is turned off in the simulator when a DTX period occurs and in non-primary cells in SSDT mode of the soft handover [9].

Interference and measurement model. The signal-to-interference ratio (SIR) is calculated in the simulator based on the instantaneous transmission powers. The partial orthogonality of the OVSF codes in the downlink is taken into account with orthogonality factor. The SIR is calculated for dedicated channels and for common pilot channel; the common pilot channel quality can be used in handover algorithms. In soft and softer handover, signal combining is modeled with selection or maximum ratio combining.

Power control. The initial transmission power for UE in the beginning of a call and for Node-B is chosen based on open-loop power control. After that, the inner-loop power control executed at every simulation step adjusts the uplink and downlink transmission powers with a fixed step based on the SIR value [10]. Transmission power limits (maximum and minimum) can be defined for Node-B. The UE maximum transmission power is dependent on the terminal type [11].

Cell selection and soft handover. The cell selection is based on common pilot channel (CPICH). The soft handover operations can be based either on CPICH Ec/Io or received power and both of these possibilities have been implemented. The link operations supported are link addition, dropping and replacement [12]. Two different algorithms have been implemented: one using relative thresholds (WCDMA as proposed in [13]) and another using absolute thresholds (no replacement operations). Also, softer handover between sectors of the same base station is supported. The signal combining in soft handover is in the uplink selection combining and in the downlink maximum ratio combining; in softer handover, the uplink combining is maximum ratio as well. Optionally, site selection diversity transmission (SSDT) can be switched on [10] influencing data channel transmission in the DL. 
Admission and congestion control framework. The framework for admission and congestion control consists of three different thresholds for load indicators: admission, start congestion and end congestion. The first threshold is used when the decision about admitting a call is made; optionally, an estimate about the effect of the new call on the load indicator can be used. The two latter thresholds are used in the congestion or load control and a timer is used with them: a cell is marked as congested in one direction if the load indicator remains above the start congestion threshold for long enough (timer) and the cell is said to be in normal state again after the load indicator has remained below the end congestion threshold long enough (timer). If the cell is congested in either direction (UL or DL), the cell is marked as congested. If any cell is marked as congested, a network level congestion status is changed to "congested".

In principle, any feasible and meaningful load indicators could be used in connection with the described framework but we support the usage of two different load indicators for both directions (uplink and downlink):

- sum of $1 / \mathrm{SF}$ (uplink and downlink)

- total transmission power of the cell (downlink)

- received interference in the cell (uplink)

In admission control, irrespective of how high the threshold values has been set, the physical resources are always checked because lack of physical resources would ultimately lead to the rejection of the call. Essential physical resources include the number of OVSF codes in the downlink and total transmission power of the cell and are thus related to two of the load indicators.

User generator and streams. The user generation and arrival in the simulator is based on defining one or multiple user streams. For each stream, the arrival intensity is given and the user arrival process is Poisson. Also, the starting time and maximum number of users can be separately specified for each user stream enabling one to create congestion peaks in time. Service type and terminal type are assigned for each user stream and thus it is possible to create e.g. such a situation, in which most of the offered traffic is speech but then at a certain instant of time some WWW users arrive at the network.

In real networks, if a user is blocked or dropped prematurely, he is likely to attempt to call again increasing offered traffic. This behavior is also modeled in the simulator by enabling one to specify the maximum number of call retries per user. The actual number of call retries is then drawn from discrete uniform distribution between zero and the given number.

Service types and QoS levels. Different service types can be defined in the simulator and a traffic model can be assigned to them. For each service type, multiple QoS levels with different parameters can be specified. 
Requested user data rates are defined in the uplink and downlink, Eb/Io requirements are given and RAB priority is specified for each QoS. The simulator keeps track of "bad quality" time of users by observing the time when the SIR is below the target value and a maximum tolerable consecutive bad quality time can be defined for each QoS level. Exceeding this results in call dropping. Also, accumulated bad quality time is recorded during simulations for each call even if it call dropping does not occur.

Traffic model. The call duration is implicitly determined by the traffic model used in the simulator. The generated traffic characteristics such as activity and asymmetry between uplink and downlink are dependent on the service type used and thus the traffic generator should be very flexible and configurable to support many kinds of service types.

The traffic generator relies on the traffic model of ETSI [14] and consists of session, packet call and packet levels. However, the same generator can be applied for generating traffic arising from e.g. speech calls by associating the packet call level to activity or inactivity periods in the speech. Thus, by changing the traffic generator parameters it can be adjusted to produce load corresponding to a wide range of different service types.

The uplink and downlink directions can be set to be coupled, which is the normal case e.g. in WWW service type where traffic in the uplink is generated in the form of a request and then the requested data arrives in the downlink direction.

The traffic generator is a relevant module for generating congestion since it has an influence on the transmission activity in the air interface, which in turn affects the interference situation in the network.

User distribution and mobility model. The user distribution and mobility model has been described in detail in our previous paper [6]. However, in this paper we are integrating that work to a more extensive simulator framework containing plenty of other features. The most relevant features are summarized here:

- arbitrary 2D user distributions can be given in a matrix

- users' mobility can be constrained to streets and the street network can be given as input in vectored format (MapInfo format)

- different mobility classes (pedestrian, vehicular) can be defined

- turning probabilities at crossroads can depends on street widths or hotspot locations

The geographical user distribution has an effect on the offered traffic load distribution between base stations and the mobility of the users influences handover rate and handover directions. Thus, different spatial congestion situations can be created using this feature.

Input and output data. Data describing the simulation scenario and parameter values are given as input to the simulator. Node-B locations, their 
neighbors and propagation losses for each cell are given in input files. The propagation losses can thus be calculated with any proper tool (e.g. raytracing). Related to the simulated congestion situation, user distribution matrix, street network, service type percentages, user stream starting times and traffic model parameters are given as input. All data defining a momentary state of the simulator is stored into number of output files enabling extensive analysis.

\section{SIMULATIONS AND CONCLUSIONS}

Three different aspects of congestion are illustrated with example simulations. The simulations shed light on different simulator features that can be used for creating various traffic load scenarios and congestion situations [8] and show how the resulting congestion situations are detected with the congestion control framework. The general simulation parameters are listed in Table 1.

Table 1. General simulation parameters

\begin{tabular}{llll}
\hline Parameter & Value & Parameter & Value \\
\hline Background noise & $-108.1 \mathrm{dBm}$ & Active set size limit & 3 \\
Congestion timer & $5 \mathrm{~s}$ & Handover timer & $1 \mathrm{~s}$ \\
Congestion start threshold & 0.3 & Add threshold (relative) & 4 \\
Congestion end threshold & 0.2 & Drop threshold (relative) & 6 \\
Power control rate & every 5 slots & Replace threshold (relative) & 2 \\
DPDCH activity, speech & 0.5 & DPDCH activity for data & 1.0 \\
Req. Eb/Io in ULDL, speech & $4.5 / 6.0 \mathrm{~dB}$ & Req. Eb/lo in UL/DL, data & $1.5 / 5.0 \mathrm{~dB}$ \\
DPCCH-DPDCH UL, speech & $3.0 \mathrm{~dB}$ & DPCCH-DPDCH UL, data & $6.0 \mathrm{~dB}$ \\
\hline
\end{tabular}

Non-uniform user distribution. In the first simulation, non-uniform user distribution created in such a way that the peak of the distribution was placed between the centers of three cells and so that the user density was 7 times as high in the peak area as in other places. The radius of the peak was 200 meters. The generated users are shown in Figure 1. The active set size for each user is also marked in the same figure with color and symbol. The average DL load indicator values from all cells and for one congested cell are shown in Figure 2. 


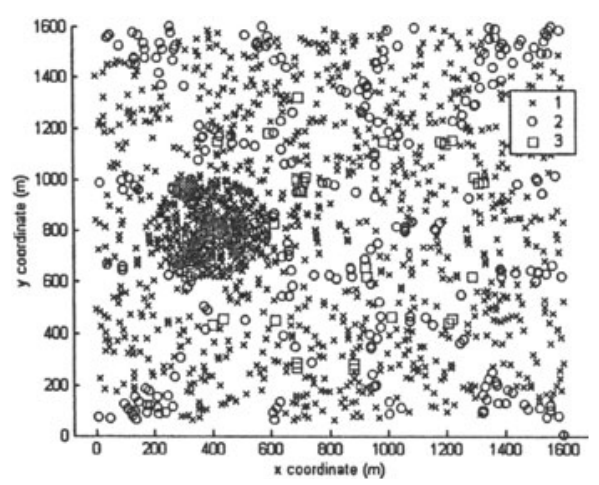

Figure 1. User locations and used active set size.

User streams. In the second simulation, two different user streams were used to create temporary congestion peak: a base stream representing constantly offered traffic and a congestion stream modeling sudden increase in the number of calls. The arrival intensity for the former stream was 1.5 and it was initialized with 100 users. In the latter stream, the arrival intensity was 3.0, it was started at 300 seconds and the maximum number of users to generate from it was 300 . The total number of users in the network and separately for each stream is shown in Figure 3. As a result of these streams, in one cell the status was changed to congested at 299 seconds and back to non-congested at 399 seconds. The load indicator only barely exceeded the start congestion threshold but since it was marked as congested, the congestion status remained as long as the load indicator dropped below the end congestion threshold for at least 5 seconds.

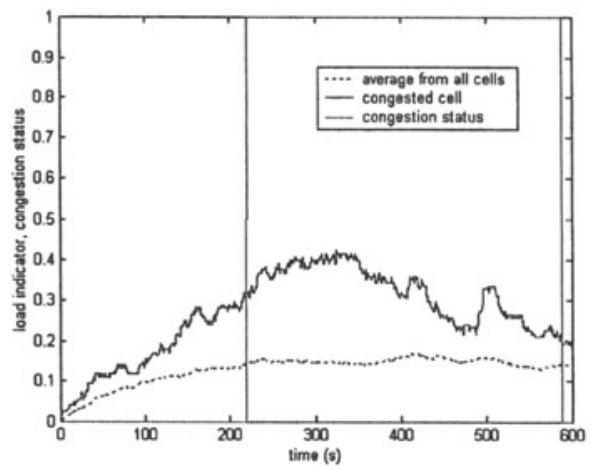

Figure 2. Average load indicator from all cells and for one congested cell. 
Service types. In the third set of simulations, two different service types were used separately: normal speech and high data rate streaming service type. The data service type used spreading factor of 16 . The initial number of users was 50. The average number of users in the simulations was 56 and 60 for speech and data, respectively. The resulting average load calculated from all cells is shown in Figure 4.

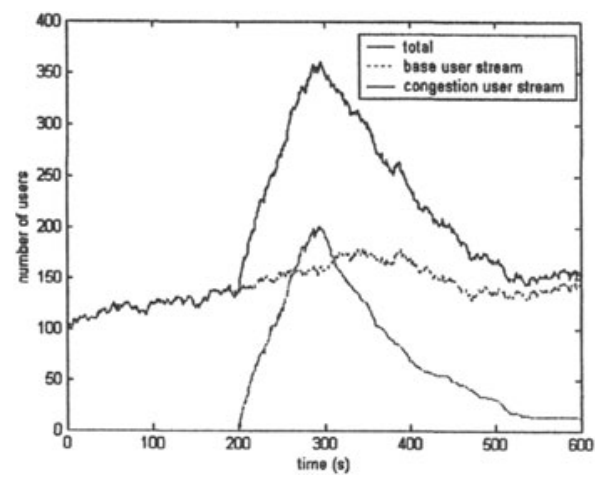

Figure 3. Total number of users and number of users in each stream.

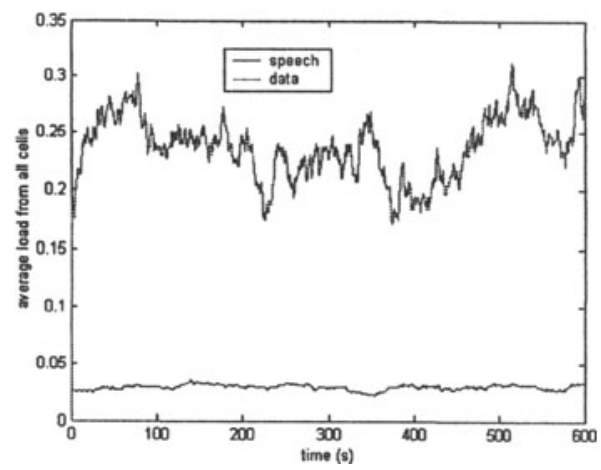

Figure 4. Average load from all cells for speech and data.

Conclusions. Based on the extensive implemented simulator core, it is possible to design and implement various resource management algorithms. The algorithms could concern changing the requested QoS levels of new calls or already ongoing calls, constraining the access to the network using access classes or prioritizing e.g. emergency calls or real-time service types using RAB priorities. Soft handover algorithms could be evaluated in 
different scenarios (different users distribution and mobility) and using either power based or SIR based handovers with absolute or relative thresholds.

Subsequent work concerning the simulator will focus on implementation of resource management techniques on top of the UMTS simulator described in this paper and evaluation of those resource management techniques.

Acknowledgements. This work has been partially performed in the framework of the project IST CAUTION, which is partly funded by the European Community. The Authors would like to acknowledge the contributions of their colleagues from National Technical University of Athens, VTT Information Technology, Cosmote Mobile Telecommunications S. A., Telia Mobile and Motorola S.p.A.

\section{REFERENCES}

[1] R. De Bernardi, D. Imbeni, L. Vignali, M. Karlsson, "Load control strategies for mixed services in WCDMA", IEEE Vehicular Technology Conference, vol. 2, pp. 825-829, Spring 2000.

[2] S. A. Ghorashi, E. Homayounvala, F. Said, A. H. Aghvami, "Dynamic simulator for studying WCDMA based hierarchical cell structures", 12th IEEE International Symposium on Personal, Indoor and Mobile Radio Communications, vol. 1, pp. 32 -37, 2001.

[3] S. Hämäläinen, H. Holma, K. Sipilä, "Advanced WCDMA radio network simulator", PIMRC, 1999.

[4] R. Hoppe, H. Buddendick, G. Wölfe, F. M. Landstorfer, "Dynamic simulator for studying WCDMA radio network performance", IEEE Vehicular Technology Conference, vol. 4, pp. 2771-2775, Spring 2001.

[5] J. Muckenheim, U. Bernhard, "A framework for load control in 3 rd generation CDMA networks", IEEE Global Telecommunications Conference, vol. 6, pp. 3738-3742, 2001.

[6] S. Nousiainen, K. Kordybach, P. Kemppi, "User Distribution and Mobility Model Framework for Cellular Network Simulations", IST Summit, Thessaloniki, Greece, 2002.

[7] W. Rave, T. Kohler, J. Voigt, G. Fettweis, "Evaluation of load control strategies in an UTRA/FDD network", IEEE Vehicular Technology Conference, vol. 4, pp. 2710-1714, Spring 2001.

[8] CAUTION IST-2000-25352, "D-3.3: Traffic Load Scenarios and Decision-Making", October 2001.

[9] ETSI, 3G TS 25.211 v 4.0.0, "UMTS; Physical channels and mapping of transport channels onto physical channels (FDD)", 2001.

[10] ETSI, 3G TS 25.214 v 4.0.0, "UMTS; Physical layer procedures (FDD)", 2001.

[11] ETSI, 3G TS 25.101 v 4.0.0, "UE Radio transmission and Reception (FDD)", 2001.

[12] ETSI, 3G TS 25.303 v 4.0.0, "UMTS; Interlayer Procedures in Connected Mode", 2001.

[13] ETSI, 3G TR 25.922 v 4.0.0, "UMTS; Radio resource management strategies", 2001.

[14] ETSI,.TR 101.112 v 3.2.0, "Selection procedures for the choice of radio transmission technologies of the UMTS", 1998. 